\title{
Les Églises dans les Cantons de l'Est (1800-1860)
}

\section{Marie-Paule Rajotte LaBrèque}

Volume 41, 1974

URI : https://id.erudit.org/iderudit/1007243ar

DOI : https://doi.org/10.7202/1007243ar

Aller au sommaire du numéro

Éditeur(s)

Les Éditions Historia Ecclesiæ Catholicæ Canadensis Inc.

ISSN

0318-6172 (imprimé)

1927-7067 (numérique)

Découvrir la revue

Citer cet article

Rajotte LaBrèque, M.-P. (1974). Les Églises dans les Cantons de l'Est (1800-1860). Sessions d'étude - Société canadienne d'histoire de l'Église catholique, 41, 87-103. https://doi.org/10.7202/1007243ar

Tous droits réservés @ Les Éditions Historia Ecclesiæ Catholicæ Canadensis Inc., 1975
Ce document est protégé par la loi sur le droit d'auteur. L'utilisation des services d'Érudit (y compris la reproduction) est assujettie à sa politique d'utilisation que vous pouvez consulter en ligne.

https://apropos.erudit.org/fr/usagers/politique-dutilisation/ 


\section{Les Églises dans les Cantons de l'Est (1800-1860)}

Parler des Cantons de l'Est, c'est aborder un sujet qui expose à beaucoup de confusion; il s'agit d'un territoire peu ou mal connu encore aujourd'hui, aux limites géographiques et administratives mal définies. On s'accorde pour y classer les cantons proches des frontières américaines, mais beaucoup refusent d'inclure dans cette région Drummondville ou Victoriaville. Pourtant, à partir des limites des seigneuries, prévaut la même tenure des terres jusqu'aux frontières et l'occupation du sol, le mode de colonisation, se déroulèrent dans des conditions semblables et dans l'espace d'une cinquantaine d'années. Période d'intense activité de défrichement, de mise en place du réseau des routes et chemins de fer, de l'organisation de la vie économique, de la création des institutions religieuses et civiles dans les agglomérations naissantes. Pour les besoins de cette étude nous proposons donc de considérer toute l'extension de ce territoire au sud-est du Saint-Laurent, entre le Richelieu et la Chaudière, non compris dans les seigneuries.

Il suffit de consulter quelques monographies régionales ou paroissiales pour connaître les terribles obstacles matériels que les colons ont dû vaincre. Ils s'attaquèrent aux forces de la nature et y déployèrent toute leur énergie; mais d'autres difficultés d'ordre psychologique et moral leur semblaient encore plus insurmontables.

En 1820, la Chambre d'Assemblée du Bas-Canada forma un comité spécial pour enquêter sur les causes du retard de la colonisation des Cantons de l'Est. Une lettre du comité adressée à tous les curés s'informait de l'opinion dans leurs paroisses respectives. Les réponses furent compilées et publiées. Si le curé Courtois de La Malbaie répond simplement que le mot Township et la chose sont inconnus chez lui, d'autres sont plus explicites. Presque tous sont rebutés par la tenure en franc et commun soccage ainsi que par les réserves du clergé et de la couronne. De plus, on recule devant les conditions d'achat qui exigent de l'argent sonnant dont bien peu disposent. Mais on insiste également sur la crainte de l'absence des secours de sa religion et de se trouver mêlés à d'autres groupes de religion et de coutumes différentes tout en vivant dans l'éloignement des parents et des amis. L'avis du curé Brodeur de 
St-Roch des Aulnets a beaucoup de résonnances chez ses confrères quand il écrit : "Nous aimerons mieux voir nos enfants dans la pauvreté ou s'établir dans nos seigneuries que de les voir puissamment riches au milieu de tels dangers et pour leur éducation et leur Religion ${ }^{1}$ ».

Malgré les sombres prévisions de ces curés, les pressions démographiques et économiques jointes à l'immigration forcèrent le peuplement des Cantons. Simultanément et de toutes les directions, ce fut l'arrivée et la progression des Américains, des immigrants écossais et irlandais et, en nombre toujours croissant, des Canadiens français.

Si quelques mécréants se sont glissés à la faveur de ce brassement de population, il faut retenir que la plupart de ces gens avaient déjà des convictions religieuses. Le problème de leur fournir les secours moraux qu'ils attendaient de leur foi et de leurs 'croyances respectives était d'une ampleur particulière. Où trouver les ressources en hommes et en argent ? Comment atteindre ces ouailles dispersées sur un territoire neuf, dépourvu de routes, sans églises, pratiquement sans écoles? Comment se faire comprendre par ces nouveaux venus qui pouvaient aussi bien ne comprendre que l'anglais ou le français ou encore que le gaélique ? Un extraordinaire mouvement missionnaire de la part de toutes les confessions a cherché à répondre à ces besoins et a suscité des dévouements et des exemples de zèle vraiment apostolique et cela, il faut le dire, chez nos frères séparés comme chez les catholiques qui nous sont mieux connus. Le prosélytisme entra bien pour une part dans ces entreprises mais ce ne fut pas l'aspect dominant; on visa surtout à maintenir ses effectifs, ce qui était vraiment le seul projet valable dans les circonstances. Des rivalités acerbes se révélèrent entre les sectes protestantes elles-mêmes et avec les catholiques à l'occasion; il ne faut pas s'en étonner quand on se rappelle les mentalités divergentes qui devaient se côtoyer. Les Américains, bien que Loyalistes ou réputés comme tels, appartenaient en général aux Églises dissidentes d'origine puritaine, méthodiste ou baptiste. Ils professaient des idées d'indépendance et refusaient le principe d'une Église d'État et d'une hiérarchie bien établie. Ils n'étaient donc pas prêts à entrer dans le giron de l'Église d'Angleterre que leurs pères avaient contestée. Leurs dispositions

1 Archives ARChev. Québec, Lettre des curés sur le défrichement des terres, 1823. 
envers les catholiques «papistes » n'étaient guère conciliantes; la Nouvelle-Angleterre subissait une propagande soutenue contre l'Église romaine qui dura pendant toute la première moitié du $\mathrm{XIX}^{\mathrm{e}}$ siècle avec virulence et qui se raviva à plusieurs intervalles ${ }^{2}$. Par ailleurs, le clergé anglican, britannique de naissance et de formation pendant de longues années, aurait voulu recréer l'atmosphère de la mère-patrie avec une Église d'État et des fidèles assidus aux offices religieux entièrement commis à la poursuite du «protestant work ethic ». Il comptait sur une suprématie fondée par l'appui du gouvernement et ambitionnait d'assumer la direction de l'éducation. Dotés d'une formation supérieure, ces hommes se permettaient d'accuser d'ignorance les prédicateurs dissidents, mais ils leur reprochaient surtout d'affaiblir la position des protestants vis-à-vis du bloc français et catholique. Bien que devant les lois le statut de l'Église catholique restait précaire, ce front uni, que la conquête n'avait pas entamé, constituait l'obstacle majeur à la réalisation des espoirs de l'Église d'Angleterre; on lui enviait son unité et sa stabilité et on comprit assez vite la futilité de toute entreprise de ce côté ${ }^{3}$.

D'autres barrières aussi intangibles mais fort contraignantes se dressaient dans le domaine légal et juridique. Dans ces nouveaux territoires non occupés sous le régime français et astreints à une tenure des terres d'origine anglo-saxonne, on s'est demandé si le droit français était valide ou si le droit anglais seul devait s'appliquer. Cette confusion devint si générale et persistante que, sur l'insistance de Georges-Étienne Cartier, le parlement du Canada-Uni légiféra pour éclaircir la situation en $1857^{4}$.

Bien que dans un régime de "tolérance octroyée », il y avait lieu de se rappeler que le traité de Paris devait «accorder aux habitants du Canada la liberté de la religion catholique(...) [et de] professer le culte de leur religion selon le rite de l'Église romaine, en tant que le permettent les lois de la Grande-Bretagne ${ }^{5}$ ». Notons

2 Robert Sylvain, La vie et l'œuvre de Henry de Courcy, Québec, 1955. Le chapitre VI présente un tableau de la position du catholicisme en Nouvelle-Angleterre à cette époque.

3 Arthur MAHEUX, "Le problème protestant 》, Rapport de la Société Canadienne d'Histoire de l'Église Catholique, 1939-40, p. 43 ss.

4 J.-C. Bonenfant, « Georges-Étienne Cartier juriste 》, Cahier des Dix, no 31, 1966, p. 19.

5 Hermann Plante, L'Église catholique au Canada, Trois-Rivières, 1970, texte cité, p. 179. 
qu'il s'agit ici d'un droit accordé aux individus et non pas à l'organisation de l'Église. La possession des biens reçus sous le régime français avait été confirmée, il y avait églises et couvents dans les paroisses des seigneuries, comment en acquérir et en établir dans les Cantons ? On rejoint l'épineux problème de l'érection civile des paroisses qui s'étendait à tout le diocèse; on n'avait encore que les directives du droit français sur les propriétés et la dîme. En attendant des législations appropriées c'est l'évêque qui devait assumer toutes les responsabilités. C'est ainsi que, le 30 juin 1818, M $^{\text {gr }}$ Plessis reçoit pour «la somme d'un chelin et autres considérations》 un terrain qui lui est cédé par le Col. Heriot pour les fins du culte à Drummondville ${ }^{6}$. De même, le 20 février $1829, M^{\text {gr }}$ Panet accepte en don de William Felton l'emplacement de la colline du drapeau (Flagstaff hill) qui demeurera le cœur de la vie catholique à Sherbrooke ${ }^{7}$. Ajoutons que les Élises dissidentes se trouvaient sur le même pied mais, en 1830, un bill autorisa toute assemblée de chrétiens ou congrégation, missions et paroisses à posséder des immeubles pour le culte et l'instruction ${ }^{8}$. La portée de cette loi fut amplifiée par la suite.

Un autre point litigieux fut la question de la célébration des mariages et de la tenue des registres en général. L'Église d'Angleterre revendiquait un monopole; la juridiction des catholiques ne fut pas contestée mais les mariages mixtes soulevèrent bien des inquiétudes dans notre clergé 9 . Ce fut moins facile pour les méthodistes et autres de se faire accepter; en 1825 on avait adopté un statut pour régulariser les actes passés par les dissidents dans le district du SaintFrançois et ce n'est qu'en 1829 que les méthodistes seront reconnus après plusieurs années d'obstruction au Conseil législatif où régnait une majorité anglicane ${ }^{10}$. Ces questions semblent assez académiques mais elles se traduisaient en situations bien concrètes pour les gens du siècle dernier pour qui les sentiments religieux et les obligations morales étaient souverains. Qu'on songe au drame que pouvait repré-

6 Fr. Côme St-German, Regards sur les commencements de Drummondville, Drummondville, 1965, p. 17.

7 Mgr Maurice O'Bready, De Ktiné d̀ Sherbrooke, Sherbrooke, 1973, p. 62 .

8 S. PAgnuelo, Études historiques et légales sur la liberté religieuse en Canada, Montréal, 1872, p. 169.

9 Nive VoIsIne, Histoire de l'Église catholique au Québec, 1608-1970, Montréal, 1971, p. 29.

10 S. Pagnuelo, op. cit., p. 164. 
senter l'impossibilité d'obtenir le baptême pour les enfants, la célébration du mariage ou la sépulture chrétienne. Pensons à tout le bagage sociologique véhiculé par le rassemblement au «meeting hall » et au ressentiment provoqué par cette forme de discrimination qui tend à humilier les convictions intimes.

Donc, vers 1830 on s'était résigné au pluralisme religieux de fait et de droit et l'Église d'Angleterre allait connaître d'autres revers qui entraîneront son déclin. Mais tout n'était pas gagné pour la paix religieuse et la facilité du culte; c'étaient encore les temps héroïques pour les colons et les missionnaires de toute allégeance.

Les évêques catholiques de Québec se préoccupaient depuis longtemps de cette région si peu accessible et dont la maigre population se concentrait près des frontières, aux environs du lac Champlain. Des ententes s'étaient établies entre les évêques canadiens et ceux des États-Unis, conférant des pouvoirs réciproques dans les deux diocèses pour desservir les fidèles qui pourraient se trouver dans ces parages éloignés. En 1806, $\mathrm{M}^{\mathrm{gr}}$ Carroll de Baltimore recevait les pouvoirs de grand vicaire de $\mathbf{M}^{\mathrm{gr}}$ Plessis et ces privilèges d'exercice étaient aussi destinés à $\mathbf{M}^{\mathrm{gr}}$ de Cheverus à Boston en $1810^{11}$. Cependant, $\mathrm{M}^{\mathrm{gr}}$ Plessis avait averti que ces arrangements ne sauraient être que temporaires; il pressentait sans doute l'inéluctable développement dont cette région serait le théâtre.

L'implantation de la vie catholique dans nos cantons a été retracée dans de nombreuses monographies. Les noms de nos grands missionnaires sont connus mais on devrait les étudier de nouveau dans leur personnalité et leurs œuvres. Tout n'a pas été dit et on trouverait beaucoup de nouvelle matière à édification, premièrement, et pour une meilleure compréhension de cette époque.

Comment les Raimbault, Holmes, Power, O'Reilley, Racine, Marquis et leurs confrères ont-ils pu jeter les bases de missions misérables, qui progressivement se transformèrent en modestes paroisses pour en arriver à former des diocèses populeux ? Il a fallu des miracles de foi et d'abnégation allant jusqu'à la mort, comme ce fut le sort de l'abbé Charles-Édouard Bélanger qui périt à 32 ans dans les savanes de Stanfold en novembre $1845^{11 a}$. Il faut reconnaître

11 Laval LAURENT, o.f.m., Québec et l'Église aux États-Unis sous Mor Briand et Mrr Plessis, Montréal, 1945, pp. 203, 204, 217.

11a Abbé Albert Gravel, Aux sources de notre histoire religieuse dans les Cantons de l'Est, Sherbrooke, 1952, p. 121. 
aussi le maintien de l'idéal chrétien du temps transmis par le catéchisme, et la tradition populaire est éloquente là-dessus. Dans les familles, la mère, les grands enfants enseignaient aux petits les prières et les questions et réponses du seul livre considéré comme indispensable ${ }^{12}$. Quelle fierté de pouvoir annoncer qu'on pouvait le réciter «de couvert en couvert ».

On connaît la pénurie de prêtres qui suivit la conquête et la situation matérielle n'était guère de nature à fournir les bases de toute une nouvelle chrétienté. C'est la générosité du clergé et des fidèles des vieilles paroisses qui fut le recours. On voit $\mathbf{M}^{\mathrm{gr}}$ Plessis ouvrir des souscriptions comme pour la construction de l'église de Drummondville en 1818 et de nouveau en 1826 à la suite d'un incendie désastreux ${ }^{13}$. Ces méthodes ne pouvaient suffire; on trouva la formule d'un organisme bien structuré dans l'Association de la Propagation de la Foi ${ }^{14}$ endossée par $\mathrm{M}^{\mathrm{gr}}$ Signay en 1836 . Cette même année vit la division du diocèse de Québec et $\mathbf{M}^{\mathrm{gr}}$ Lartigue devenait évêque avec pleins pouvoirs dans l'ancien district de Montréal. Une partie des cantons, jusqu'à Hatley et Barford, passaient alors sous sa juridiction et il ne tarda pas à se pourvoir aussi de l'instrument de la Propagation de la Foi. Bien entendu, ce n'était pas seulement pour ses «Townships » mais notre région en a obtenu beaucoup de bienfaits surtout du temps de $\mathbf{M}^{\mathrm{gr}}$ Bourget. Les Rapports de ces deux sociétés ${ }^{15}$ nous offrent le tableau des missions des Townships dans ces années de croissance. Il arrive que les récits de nos missionnaires semblent ternes et laconiques auprès des épopées qui décrivent les équipées de l'Orégon, de la Rivière-Rouge, du Témiscamingue, de Wala-Walla; nous n'étions pas les seuls dans le besoin. Mais aux accents de rapports des visites pastorales chez nous, des lettres citées, on sent la sollicitude profonde et la compassion pour nos misères. Voici des extraits de la relation de la première visite de $\mathrm{M}^{\mathrm{gr}}$ Bourget dans les Cantons de $1^{\prime} E s t$ en 1842; à travers le style de l'époque perce tout le cœur du pasteur attentif :

Nous avons visité les catholiques dispersés dans les Townships de l'est. Depuis longtemps [ils se plaignaient de l'abandon et il était] impossible alors d'aller à leur secours (...). Nous commençames le trente juillet dernier cette visite après laquelle nous soupirions depuis si longtemps. (...) Vous raconter ici tout ce que firent les catholiques de ces lieux pour nous montrer

12 Fernand PORTER, o.f.m., L'Institution catéchistique au Canada, Montréal, 1949, p. 209 ss. 
leur joie; vous dire avec quel zèle ils quittèrent tout pour suivre les exercices de la visite; vous exprimer la violence qu'il nous fallait nous faire pour nous arracher à leurs larmes et gémissements quand nous avions terminé notre visite, c'est ce que ne nous permettent pas les bornes de cette lettre pastorale.

Les catholiques de ces missions sont généralement pauvres et la plupart obligés de louer leurs services à des maîtres ennemis de leur religion (...) [Plusieurs] voulurent les empêcher d'assister aux exercices de la Visite Pastorale. (...) Nous devons pourtant à la justice de dire qu'il s'en faut que tous nos frères séparés aient montré le même acharnement contre de pauvres catholiques, qui n'ont d'autre bien en ce monde que leur religion et qui veulent à tout prix le conserver. Car nous avons eu la consolation de voir que nombre d'entre eux ont été assidus aux instructions; (...) Il fesait (sic) beau voir ces frères encore séparés de nous par des erreurs que nous déplorons de tout notre cœur, se mettre à genoux, pour baiser et recevoir avec respect les images de la Tempérance. (...) Nous devons vous dire pour votre édification que nos travaux ont été heureusement secondés par plusieurs personnes du monde qui s'étaient portées avec un zèle admirable à instruire les ignorans, pour les préparer d'avance à bien recevoir les sacrements. (...)

Nous avons nommé deux prêtres qui vont sans cesse visiter ces lieux afin d'y achever une œuvre si heureusement commencée. Nous espérons que ces visites contribueront non seulement au bien spirituel des catholiques de ces lieux reculés mais encore à leur bien temporel. Du moins allonsnous prendre des arrangements pour atteindre ce double but que nous nous proposons, savoir la prospérité spirituelle et temporelle de notre peuple 16.

$\mathrm{M}^{\mathrm{gr}}$ Bourget laisse présager le sens éminemment réaliste qu'il entend donner à son action qui se traduira concrètement par ses

13 C. St-Germain, op. cit., p. 18.

14 Lionel Groulx, Le Canada missionnaire, Montréal, 1962, p. 18.

15 Pour le diocèse de Montréal, voir : Robert VaLors, «Étude bibliographique sur les Rapports de l'Association de la Propagation de la Foi à Montréal », Revue d'histoire de l'Amérique française, vol. IV, mars 1951, pp. 560-567 et vol. V, pp. 307-308. Dans le diocèse de Québec, les quatre premiers numéros portent le titre de Notice sur les missions..., etc., par la suite, Rapport sur les missions...

16 Rapport de l'Association de la Propagation de la Foi, Montréal, no 4, déc. 1842, pp. 6-7. 
apports aux œuvres de colonisation. D'autres interventions s'ajoutent; l'abbé Bernard O'Reilly étale dans les journaux, à partir de 1848, la situation intenable des colons des Townships, puis, le «Manifeste des douze missionnaires » Le Canadien Émigrant, en 1851, obtint un retentissement mérité. Ces documents ne se contentaient pas d'exposer des doléances, ils proposaient des solutions. Sans doute mis en éveil par l'abbé O'Reilly, l'Institut Canadien de Montréal s'était donné une vocation d'animateur et, dans un élan d'enthousiasme sans précédent, on lança une «Association des Établissements Canadiens dans les Townships » en avril $1848{ }^{17}$. Il semble difficile de croire aujourd'hui que $\mathbf{M}^{\mathrm{gr}}$ Bourget en avait accepté la présidence; mais il tenait tant au progrès de cette région. Malheureusement, l'intrigue politique noyauta rapidement l'organisation qui échoua. Toutefois, $\mathrm{M}^{\mathrm{gr}}$ Bourget avait eu le temps de favoriser la fondation d'une colonie à Roxton Falls que son coadjuteur avait inaugurée avec panache en donnant le premier coup de cognée sur l'emplacement de la future église. L'évêque de Montréal dut faire face aux obligations contractées avec l'aide de la Propagation de la Foi ${ }^{18}$, mais instruit par cette aventure il créa avec les autres évêques une nouvelle Association pour la Colonisation des Townships en 1850. Sans détour, il en présente les objectifs dans une lettre pastorale :

(...) Nous avons à fixer sur notre sol les domestiques de la foi, nos propres concitoyens. Un des moyens les plus efficaces est de faire marcher à la tête des colons le Prêtre, la croix à la main. Il faut à ce peuple colonisateur des temples et des ministres du Seigneur. Mais il est pauvre et dans les commencements qui en fera les frais? La Société de la Propagation de la Foi. Il faut aussi à ces jeunes colons sans expérience de bons guides, où les trouver? Dans le Conseil central, car il se composera d'hommes religieux et patriotes prêts à faire servir connaissances et influence dans la société à l'avantage des nouveaux habitants des Townships 19.

Qui ne reconnaîtra ici le modèle de la société pastorale, loin de la contamination des villes, sous la houlette de pasteurs sages et bienveillants. Tout de même on ne dédaignera pas un brin d'influence

17 Léon Pouliot, s.j., M ${ }^{g r}$ Bourget et son temps, Montréal, 1972, t. III, 2e partie, p. 46.

18 Rapport de l'Association de la Propagation de la Foi, Montréal, 1850 , avant-propos.

19 Ibid., janvier 1851, p. 5 . 
laïque... pour épauler le missionnaire colonisateur dont l'ère va durer un siècle.

1852 marque un autre aménagement des diocèses du territoire. Trois-Rivières enlève à Québec les régions du bas Saint-François (destinées à former le diocèse de Nicolet en 1885), tandis que les autres, avec la ville de Sherbrooke sont englobées par Saint-Hyacinthe. La prépondérance catholique et française est maintenant assurée dans ces townships mais un terrain demeure en friche, celui de l'éducation; nous y reviendrons.

Pendant toute cette période, le protestantisme dans nos parages est loin d'être une institution monolithique. Certes, l'Église anglicane semble grandement favorisée par son statut apparent d'Église d'État et l'appui de solides sociétés missionnaires de la mère-patrie, entre autres, la Society for the Propagation of the Gospel in Foreign Parts, fondée en 1701, et la Society for the Propagation of Christian Knowledge plus ancienne de deux ans. Pourtant, elle n'a pas le privilège d'avoir été la première à occuper le terrain.

Nous avons vu que les colons d'origine américaine avaient importé leurs croyances particulières; l'organisation très souple de leur culte s'adaptait parfaitement aux conditions de vie des commencements d'une colonie. Un clergé plus ou moins autodidacte, parfois assez pittoresque, parcourait les établissements dispersés; un itinéraire donné s'appelait un circuit et dans une région plus vaste on les regroupait en conférences. Dans bien des cas, la Bible était le seul bagage théologique et on y puisait inlassablement pour chacune des quatre visites annuelles que le révérend devait faire dans son circuit. Dès la première heure des hommes d'une formation intellectuelle plus avancée se présentèrent et établirent des groupements plus stables. C'est le Rev. William Marsh qui établit une église Baptiste à Sutton en 1796 qui devint le noyau d'un circuit qui s'étendit à Brome, Stanstead, Hatley, Compton, Ascot. Comme pour les autres sectes, c'est la guerre de 1812 qui coupera les relations suivies avec les Etats-Unis d'où venait la principale source du personnel et de l'appui matériel ${ }^{20}$. C'est à Farnham seulement que les Quakers firent souche; Aaron Bull, arrivé dès 1801, vit prospérer

20 Stuart Ivison, The Baptists in Upper and Lower Canada before 1820 , Toronto, 1956, p. 160. 
son établissement et en 1835 on y érigeait un «meeting house » à côté d'un petit cimetière ${ }^{21}$.

C'est aussi avec l'appui américain que les Congrégationalistes se réunirent à Rock Island; leur ferveur était si grande qu'ils se contentèrent d'une grange pour se rassembler mais ils connurent des jours meilleurs et une puissante société anglaise 22 leur accorda son patronage. L'apport le plus important à la vie religieuse des dissidents fut fourni par les méthodistes. Le type du pasteur itinérant est resté légendaire et il fallait vraiment être d'une trempe particulière pour mener semblable existence. Ils ne furent pas tous des excentriques, mais quelques-uns sont restés mémorables. Par exemple, le rév. Lorenzo Dow, du circuit de Dunham et Sutton en 1799, qui était si peu ponctuel dans ces tournées qu'on le comparait à une comète dont on ne sait jamais quand elle reparaitra; par contre on appréciait ses visites et on ne se plaignait pas qu'elles fussent trop longues ${ }^{23}$. Il n'était pas rare que les pasteurs prolongeaient leurs sermons pendant plusieurs heures et si les hôtes étaient trop pauvres pour leur offrir à manger, ils reprenaient la route sans plus. Un autre, Peter Vannest, pourchassait la vanité jusqu'au point de défendre les boutons sur les habits et les faire remplacer par des agrafes qu'il jugeait plus modestes; son zèle, assez fanatique, le soutenait dans les centaines de milles qu'il couvrait à pied par tous les temps. Au moment de la guerre de 1812, tous ces prédicateurs américains restèrent chez eux sauf le jeune Robert Hibbard qui avait organisé le circuit de la Rivière Saint-François qui semblait très prometteur. Envoyé dans la région de l'Outaouais, il décida de revenir à son ancien poste quand il apprit que tous les circuits des Townships avaient été désertés, mais, en cours de route, il se noya en traversant le fleuve près de Montréal ${ }^{24}$.

La paix revenue, les activités des méthodistes américains reprirent mais pas pour longtemps dans le Bas-Canada. Des missionnaires Wesleyens envoyés d'Angleterre provoquèrent de sérieux différends et, après 1820, une entente leur réserva le champ libre; seul le

21 A.G. DoRland, The Quakers in Canada, a History, Toronto, 1968, p. 38.

22 E.C. Woodley, Old Quebec Trails and Homes, Toronto, 1946, p. 102.

23 George Playter, History of Methodism in Canada, Toronto, 1862, p. 61 .

24 Ibid., p. 112. 
Haut-Canada conserva ses affiliations américaines pendant quelques années encore ${ }^{25}$.

Avec des moyens bien modestes et animés de grande sincérité, les représentants de ces diverses confessions apportèrent des consolations religieuses à des hommes et des femmes dont l'isolement était l'un des grands maux. Ils furent ridiculisés par des anglicans comme Mountain et Strachan, diplômés d'Oxford, mais ils eurent le mérite de maintenir un minimum de vie spirituelle et d'inspirer quelques bons sentiments au moment où personne d'autre ne pouvait le faire.

Dans l'esprit de certains administrateurs anglais, les Townships de l'est étaient destinés à recréer une petite Angleterre 26; la tenure des terres, la toponymie et la religion devaient y contribuer. Par d'importantes concessions de terre à des particuliers, on avait espéré constituer une «landed gentry» qui serait un admirable élément de stabilité et de vie civilisée. Ces intéressantes personnes ne daignèrent pas s'établir dans leurs domaines; il resta le clergé et il fut de qualité. À partir de Jacob Mountain, évêque du XVIII siècle d'une Église établie qui se vit contraint à une vie de luttes ingrates, contre ses propres amis, souvent, pour tenter d'imposer l'autorité dont il se croyait investi ${ }^{27}$. Il ne recula pas devant les obligations de sa tâche apostolique et visita son immense diocèse à plusieurs reprises. C'est en 1815 qu'il traverse nos cantons pour la première fois dans des conditions qu'on imagine : dormant sous la tente, s'estimant chanceux de parcourir une douzaine de milles par jour à travers maints obstacles, et cela à 66 ans ${ }^{28}$. Son fils George, qui sera aussi évêque de Québec, doit un soir d'hiver coucher dans une cabane de colon où le froid l'obligera à garder son manteau de castor et il tentera de boucher les trous d'une fenêtre avec ses bas ${ }^{29}$. Ces misères étaient le partage de tous à ce qu'il semble. Mais le grand apôtre de l'Église d'Angleterre dans nos régions fut le

25 Ibid., pp. 150, 234.

26 Fernand Ouellet, Histoire économique et sociale du Québec, 17601850, Montréal, 1966, p. 156.

27 C'est un de ses amis, Herman W. Ryland, qui écrit en 1804 à une personne non identifiée : «My creed, with regard to the Protestant Church establishment in these Provinces, is simply this : it ought to have as much splendour and as little power as possible(...). »Cité par John MorR dans Church and State in Canada, 1627-1867, Toronto, 1967, p. 121.

28 A. Mountain, A Memoir of George J. Mountain, Montréal, 1866, p. 34.

29 Ibid., pp. 43-44. 
Rév. Charles James Stuart, qui a laissé un grand renom de sainteté par son zèle et sa charité. De haute naissance, fils du comte de Galloway, allié à de grandes familles de son pays natal, il avait désiré se consacrer à l'évangélisation des Indiens malgré une constitution faible. Arrivé à Québec, on ne le prit pas trop au sérieux et il accepta de bon gré de changer sa destination pour Saint-Armand. Il passa une dizaine d'années à Frelighsburg et à Hatley où il se fit le promoteur, et le bailleur de fonds, pour la construction de plusieurs églises. Il fut aussi l'instigateur d'un petit collège théologique à Chambly qui, après bien des vicissitudes, conduisit à l'établissement de Bishop's à Lennoxville. Devenu évêque de Québec, son expérience missionnaire lui fut précieuse. Partisan de la modération, il se refusa toujours à s'attaquer à l'Église catholique ${ }^{30}$. Plusieurs autres clergymen ont laissé un souvenir vivace, comme le Rév. Cotton de Dunham et le Rév. Woods de Drummondville et Trois-Rivières, éducateur estimé qui fut même pressenti comme principal de McGill lors de son organisation effective ${ }^{31}$.

Comment vivait cette Église d'Angleterre avec un clergé relativement peu nombreux et des fidèles encore clairsemés? On les croyait riches et ils étaient certes les mieux pourvus. Cette énorme dotation que constituaient les réserves du clergé semblait une garantie d'amples ressources. On sait aujourd'hui que ce ne fut pas le Pactole espéré et que dès le départ, la planification en était vouée à l'échec. L'administration des fonctionnaires, les conditions politiques et économiques firent le reste. En 1839, après la vente de 300000 acres, on percevait un revenu annuel de $f 942$ seulement. L'important salaire du titulaire du diocèse, £2000 plus allocations, était défrayé par le gouvernement ${ }^{33}$. Au début un missionnaire retirait £200 par année avec résidence fournie; une grande partie de ces fonds provenait des sociétés missionnaires S.P.G. et S.P.C.K. dont la dernière défrayait en partie le coût de construction des

30 T. Millman, The Life of the Right Rev. the Honourable Charles James Stewart, London (Ont.), 1953, p. 168.

31 James H. Lambert, «The Reverend Samuel Simpson Wood, BA, MA : A Forgotten Notable, and the Early Anglican Church in Canada », Journal of the Canadian Church Historical Society, vol. XVI, n ${ }^{\circ}$ 1, March 1974, p. 8.

32 T. Millman, op. cit., p. 109.

33 Les octrois du gouvernement passaient par la caisse de l'armée qui couvrait aussi l'allocation de $£ 1000$ à l'évêque catholique, ainsi que $£ 600$ à l'Église d'Écosse : Mrllman, op. cit., p. 114. 
églises. Cependant ces sociétés émargeaient aussi au budget de la couronne britannique en plus de recevoir des dons des particuliers; les largesses furent restreintes quand le gouvernement retira graduellement son apport de 1831 à 1835 . Les salaires tombèrent à $£ 100$ en moyenne, ce qui était assez mince pour un clergé marié et chargé de famille. De nouveau, la Vénérable société réduisit son assistance au Canada en 1855, ne pouvant suffire à pourvoir tout l'Empire britannique en pleine expansion. À titre d'exemple, pour l'année 1828, la S.P.G. avait fourni dans les deux Canadas la somme de $\mathbf{f} 9774$ pour l'entretien de 58 missionnaires, 8 maîtres d'école et 5 étudiants en théologie ${ }^{34}$. On peut donc juger de l'importance vitale de ces organismes pour le développement de l'Église anglicane dans un pays nouveau comme le nôtre. À mesure que les secours de l'extérieur diminuaient, la mesure d'autonomie locale s'accroissait; on peut dire que l'Église se canadianisait et se rapprochait des réalités locales et contemporaines tout en gardant la nostalgie de l'ordre ancien.

Malgré des auspices favorables pour une chrétienté protestante dans les Cantons de l'Est, c'est le contraire qui s'était produit. George Mountain dresse un bilan réaliste dans un rapport rédigé au cours d'une visite épiscopale en 1861.

(...) There are many circumstances attaching to the whole field of labour in the Eastern Townships which present an aspect of discouragement and difficulty. Wherever there is a predominance or a considerable infusion of settlers who are American or of American descent there is a great proportion of the population who, drawing their origin from a country in which there are no national religious institutions, are unconnected with any religious organization whatever, and who remain unbaptized, and, in a vast number of instances, totally regardless of the duty of attending public worship. And, in what does appear in the shape of Religion, there is frequently to be witnessed a frothy torrent of extravagant fanaticism which is apt to run itself speedily dry, as well as a multiform exhibition of doctrinal error, (such as is propagated by the Universalists, the Adventites, the seventh-day Baptists, \&c.) serving, altogether, to bewilder the minds of the people and to make many stand aloof from Religion. (...)

The Roman Catholic Church has of late years made great advances in these townships not, thus far, in the way of

34 T. Millman, loc. cit. 
proselytism, but by means of accessions which it affords to the population year by year, tending, in the probable result, numerically to overbalance the Protestants in portions of the country heretofore considered as distinguishingly Protestant, and to gain the ascendency in matters of municipal concern. The vast facilities afforded in the formation of certain new settlements by the Government, and the large advantages there opened to the settler, appear to be so dispensed and administered as eminently, - I believe I should not be far wrong in saying exclusively, - to favor the Roman Catholic interest ${ }^{35}$.

L'évêque Mountain avait vu juste et l'analyse du document révèle ses principaux soucis mais les tendances étaient irréversibles et le rapport des forces avait changé pour toujours.

Malgré l'avance perceptible des catholiques, il faut constater une grande faiblesse dans un secteur, celui de l'éducation dans nos cantons. Au moment où $\mathrm{M}^{\mathrm{gr}}$ Prince assume la charge du nouveau diocèse de Saint-Hyacinthe, il s'en fait une priorité. En 1853 il obtient une modeste subvention de $\$ 240$. de la Propagation de la Foi pour des écoles à Sherbrooke. Dans sa lettre de remerciement il dit bien que le double n'aurait pas été de trop car cette paroisse est peut-être la plus démunie de son diocèse avec la plus vieille chapelle, totalement insuffisante pour la population. «Il n'y a pas une bonne école catholique et les protestants en ont par demidouzaines ${ }^{36}$. 》 Soulignons ici tout le problème des mentalités et des institutions .

L'instruction pour tous n'était guère une préoccupation générale sous l'ancien régime. Marguerite Bourgeois avait été une innovatrice notoire dans son temps avec ses petites écoles populaires; l'éducation était encore réservée à une élite. Après la conquête, tout était à faire et nos évêques ont multiplié les efforts en ce sens. $M^{\text {gr }}$ Plessis recommande aux curés d'établir des écoles dans leurs paroisses ${ }^{37}$,

35 Quebec Diocesan Archives, G-14, Occasional Papers of the Church Society of the Diocese of Quebec, p. 13.

36 Notes historiques sur le diocèse de Sherbrooke, livre formé de fascicules divers provenant de publications non-identifiées, sans lieu ni date, peut-être du Séminaire de Sherbrooke. Pagination discontinue.

37 «Inventaire de la correspondance de Mgr. J.O. Plessis», Rapport des Archives de la Province de Québec, 1927-28, p. 285. Parmi tant d'autres, un exemple du zèle pratique de l'évêque : à $\mathbf{M}$. $\mathbf{H}$. Vallée, curé à St-Pierredu-Sud, «... Insistez souvent dans vos sermons, mon cher curé, sur les avan- 
il soutient la fondation des collèges de Nicolet et de Saint-Hyacinthe. $\mathrm{M}^{\mathrm{gr}}$ Lartigue salue l'arrivée des FF. des Écoles Chrétiennes en 1837 qui vont "répandre dans le diocèse une éducation chrétienne et solide pour les garçons comme nous en avons déjà une pour les filles par le moyen des Srs de la Congrégation établies en Canada dès le commencement de cette colonie $37 a$ ». On connait le développement des instituts enseignants dans les décennies suivantes.

Par contre les immigrants américains avaient une longue tradition de scolarisation, à partir de l'élémentaire. Dès 1642, les Puritains du Massachusetts créaient l'obligation d'instruire les enfants pour qu'ils puissent lire la Bible et comprendre les principales lois du pays ${ }^{38}$. L'école était le premier édifice communautaire qu'ils construisaient. Ils se comportèrent de la même manière ici. Par exemple, à Shipton (aujourd'hui Richmond), l'école bien modeste est construite en 1807, mais déjà une demoiselle enseignait dans une maison privée depuis 1801; on connaissait déjà le concept d'éducation permanente puisque les colons s'organisaient une bibliothèque circulante en 1815 , la Craig Union Library ${ }^{39}$. Sutton avait aussi son école en 1808 , et en février 1816 , quelques mois à peine après la fondation de Drummondville, le Col. Heriot annonce qu'on se prépare à construire une école ${ }^{40}$.

Les luttes contre l'Institution royale ne se limitèrent pas à l'opposition des catholiques. Dans les Cantons de l'Est, les Loyalistes, Écossais et autres, affirmèrent aussi leur méfiance à l'égard d'un système dominé par un évêque anglican ${ }^{41}$. Pendant longtemps, même après l'Union des Canadas, ils continuèrent à réclamer avec insistance l'enseignement de la Bible dans toutes les écoles ${ }^{42}$. Par contre, on leur reprochait leurs méthodes et leurs manuels par trop répu-

tages qui peuvent résulter de l'éducation (...) et répétez souvent aux paroissiens que, quand même ils n'auraient pas le dessein de faire étudier le latin à leurs enfants, il serait encore à-propos qu'ils leur fissent apprendre à lire, à écrire, à compter... 》 (14 nov. 1811).

37a Ibid., 1945-46, «Correspondance de Mgr. Lartigue 》, lettre au Fr. Anaclet, sup. des F.E.C. (2 janv. 1838), p. 343.

38 E.C. WOODLEY, op. cit., p. 90.

39 Annals of Richmond County and Vicinity, Richmond, 1966, vol. I, p. 47.

40 C. St-Germain, op. cit., p. 13.

41 K.D. Hunte, The Development of the System of Education in Canada East, 1841-67, Montréal, 1963, Thèse Univ. McGill, pp. 16, 78.

42 Ibid., p. 129. 
blicains ${ }^{43}$. L'animosité entre anglicans et dissidents fut telle qu'en visant les influences américaines, on chercha à proscrire tous les étrangers de l'enseignement ${ }^{44}$. Du renfort pour les écoles de l'Église d'Angleterre se présenta sous la forme de la Newfoundland and British North American School Society accueillie à bras ouverts par l'évêque George Mountain ${ }^{45}$, même si à Terre-Neuve l'évêque Feild avait eu avec elle des différends d'ordre théologique, la Société étant nettement d'orientation évangélique, tandis que l'évêque penchait vers les Tractariens ${ }^{46}$. Ces querelles de High et Low church n'eurent pas de répercussions trop fâcheuses par ici et c'est à Sherbrooke que cette société vint ouvrir sa première école en 1839. Deux enseignants, $\mathbf{M}^{\mathbf{r}}$ et $\mathbf{M}^{\mathbf{r s}} \mathrm{King}$, s'installèrent dans une bâtisse fournie par la British American Land Co. ${ }^{47}$.

Autre facette du problème scolaire, les catholiques se trouvaient en minorité dans les conseils; seuls les propriétaires y étaient éligibles et les protestants en occupaient massivement les sièges. En 1854 , la somme de $\$ 600$. octroyée par la Législature pour les écoles de l'endroit avait abouti au conseil scolaire du canton d'Ascot qui restait sourd aux réclamations des catholiques français. L'année suivante, deux commissaires canadiens-français se faisaient élire et en 1856 on ouvrait une classe française tenue par la søur du curé Dufresne 48. C'est ici qu'on comprend mieux l'inquiétude de George Mountain au sujet de l'influence des catholiques au niveau des institutions municipales.

Quand le diocèse de Sherbrooke deviendra une réalité en 1874, chacun aura pris la mesure de ses forces et de ses possibilités. Le préambule de la première Notice de la Propagation de la Foi de Québec, 1839, énumérait les buts de la Société : entre autres bonnes œuvres, on devait « imprimer et diffuser des catéchismes en langue française et anglaise ainsi que quelques autres livres de religion et de controverse parmi nos frères catholiques qui sont dans les Townships et autres lieux qui sont privés de la résidence de

43 Ibid., p. 41.

44 Ibid., p. 81. On faisait exception pour les Frères.

45 Ibid., p. 45.

46 Fred. Jones, "The early opposition to Bishop Feild of Newfoundland », Journal of the Canadian Church Historical Society, vol. XVI, no 2 , June 1974.

47 K.D. HunTE, op. cit., p. 41.

48 A. Gravel, op. cit., p. 38. 
prêtres ${ }^{49}$ ». Nous ne connaissons pas l'usage ni l'effet de ces "livres de controverse » et nous croyons que les catéchismes ont joué un rôle irremplaçable dans le maintien de la foi populaire. Il est peu probable qu'ils aient contribué à beaucoup de conversions si ce n'est au sein de familles unies par les liens de mariages mixtes, et encore, c'est une hypothèse. Par ailleurs, les défections de catholiques furent relativement rares et tinrent surtout de chicanes locales de bancs ou de clochers, d'emplacements de chapelles ou de conflits de personnalité.

Cette brève esquisse ne peut que faire soupçonner toute la complexité de l'histoire religieuse des Cantons de l'Est. Elle est remplie de personnalités fortes et attachantes; par le pluralisme de ses premiers colons elle offre un champ d'étude qui peut fournir beaucoup de données originales. Qui sait, on pourrait peut-être cerner mieux ensuite ce type un peu mystérieux: l'homme des Cantons de l'Est.

Mme Marie-Paule R. LABRÈQue, M.A., Université Laval, Québec.

49 Notice sur les missions du diocèse de Québec qui sont secourues par l'Association de la Propagation de la Foi, Québec, $\mathrm{n}^{\circ}$ 1, janvier 1839, Avantpropos. 